\section{INFLAMMATION}

\section{IL-1 signalling in atherosclerosis}

The $\alpha$ and $\beta$ isoforms of IL- 1 have differential effects on atherosclerosis, according to new experimental data. These findings are important to consider when selecting anti-inflammatory therapies for patients with cardiovascular disease and are particularly relevant in the context of the CANTOS trial.

During early atherogenesis in $\mathrm{Apoe}^{-/-}$ mice fed a Western diet, treatment with monoclonal antibodies against IL-1 $\alpha$ or against both IL-1 isoforms decreased atherosclerotic lesion area and compensatory outward remodelling in the aortic root, whereas antibodies against IL- $1 \beta$ did not impair outward vessel remodelling. In hypercholesterolaemic Apoe $e^{-/-}$mice with established atherosclerosis, selective IL-1 $\beta$ neutralization promoted a shift in circulating monocytes to a less inflammatory state, increased the levels of circulating IL-10 (an anti-inflammatory cytokine) and decreased the area of established atheromata in the aortic root, without limiting outward vessel remodelling. By contrast, IL-1a neutralization had no significant effect on established atheromata. These findings indicate that IL-1 $\alpha$ is involved in early atherogenesis, whereas IL-1 $\beta$ drives inflammation during the evolution of advanced atheroma.

The investigators suggest that the observed differences might arise from distinct signalling mechanisms of the two isoforms. IL- $1 \alpha$ is mainly associated with the cell membrane and operates via juxtacrine signalling, whereas IL- $1 \beta$ is released and acts through paracrine and endocrine signalling.

IL-1 signalling can be inhibited clinically with the use of canakinumab (a monoclonal antibody against IL-1 $\beta$ ) or anakinra (an antagonist of the IL-1 receptor that blocks the action of both IL-1 isoforms), and an antibody against IL-1 $\alpha$ has undergone clinical testing. Moreover, the CANTOS trial showed that canakinumab reduced recurrent cardiovascular events in patients with myocardial infarction who had residual inflammatory risk. The current experimental findings help to interpret the CANTOS trial and to guide anti-inflammatory interventions in patients with cardiovascular disease.

Gregory B. Lim

ORIGINAL ARTICLE Vromman, A. et al. Stage-dependent differential effects of interleukin- 1 isoforms on experimental atherosclerosis. Eur. Heart J. https://doi.org/10.1093/eurheartj/ ehz008 (2019)

\title{
Mobilizing regulatory $T$ cells to promote myocardial repair
}

Mobilization of regulatory $\mathrm{T}\left(\mathrm{T}_{\text {reg }}\right)$ cells from the spleen to the site of myocardial infarction can be promoted with the use of a CXC-chemokine receptor 4 (CXCR4) blocker, and the infiltrated $\mathrm{T}_{\text {reg }}$ cells attenuate inflammatory gene expression in monocytes and macrophages and augment infarct repair. These findings come from a study in mice and pigs published in Circulation.

Intraperitoneal injection of the peptide macrocycle POL5551, a CXCR4 antagonist, into wild-type mice with reperfused myocardial infarction increased angiogenesis in the border zone of the infarct and decreased scar size, left ventricular remodelling and contractile dysfunction at 28 days. Administration of POL5551 was shown to mobilize $\mathrm{T}_{\text {reg }}$ cells into the peripheral circulation, followed by accumulation of $\mathrm{T}_{\text {reg }}$ cells in the myocardial infarct region. The treatment effects of POL5551 were absent in splenectomized wildtype mice, $\mathrm{T}_{\text {reg }}$ cell-depleted DEREG mice and lymphocyte-deficient Rag1-knockout mice. In the infarct zone, the increased presence of
$\mathrm{T}_{\text {reg }}$ cells resulted in attenuated expression of inflammatory genes in monocytes and macrophages.

To test the translational potential of this approach, the researchers used a pig model of reperfused acute myocardial infarction. Intravenous injection of POL6326 (a clinicalstage analogue of POL5551) decreased infarct volume and improved left ventricular ejection fraction, measured using serial contrast-enhanced MRI.

"These findings should stimulate further research into the therapeutic potential of CXCR4 blockade after myocardial infarction and in other acute conditions wherein excessive innate and/or adaptive immune responses cause immunopathology," conclude the researchers.

Gregory B. Lim

ORIGINAL ARTICLE Wang, Y. et al. C-X-C motif chemokine receptor 4 blockade promotes tissue repair after myocardial infarction by enhancing regulatory $T$ cell mobilization and immune-regulatory function. Circulation https://doi.org/ 10.1161/CIRCULATIONAHA.118.036053 (2019)

\section{DYSLIPIDAEMIA}

\section{Statin efficacy in primary CVD prevention might diminish with patient age}

Statin therapy significantly reduces major vascular events irrespective of age, but less direct evidence is available on the benefit of statins for the primary prevention of cardiovascular disease (CVD) in people aged $>75$ years. These observations come from a meta-analysis performed by the Cholesterol Treatment Trialists' Collaboration.

The meta-analysis included 28 randomized, controlled trials on statins involving a total of 186,854 participants, of whom $8 \%$ were aged $>75$ years at randomization. For each $1 \mathrm{mmol} / \mathrm{l}$ reduction in LDL-cholesterol level, statins reduced the overall risk of major vascular events by $21 \%$ and vascular death by $12 \%$. The proportional reduction in major vascular events was slightly smaller with increasing age, but the trend was not significant. Smaller proportional reductions in vascular death with age were also observed $\left(P_{\text {trend }}=0.004\right)$, but the trend did not persist after exclusion of four trials on patients with heart failure or who were receiving renal dialysis, in whom statin therapy has little or no benefit.
The proportional reductions in major coronary events diminished with age $\left(P_{\text {trend }}=0.009\right)$ and persisted after exclusion of the heart failure and dialysis trials.

Interestingly, although the proportional reduction in major vascular events was similar irrespective of age in patients with a history of vascular disease (that is, in secondary prevention), the proportional risk reductions were smaller in older than in younger participants with no known vascular disease $\left(P_{\text {trend }}=0.05\right)$. The investigators speculate that this trend might be due, among other reasons, to a reduced capacity for statins to affect advanced atherosclerosis and to greater diagnostic uncertainty at older ages. Trials to assess statin therapy for the primary prevention of CVD in old people are ongoing. Irene Fernández-Ruiz

ORIGINAL ARTICLE Cholesterol Treatment Trialists' Collaboration. Efficacy and safety of statin therapy in older people: a meta-analysis of individual participant data from 28 randomised controlled trials. Lancet 393, 407-415 (2019) 